\title{
Year in review 2014: basic science and epidemiology
}

\author{
Clare Lloyd, Paul Cullinan
}

Imperial College (National Heart and Lung Institute), London, UK

\section{Correspondence to} Dr Paul Cullinan, Imperial College (National Heart and Lung Institute), London SW3 6LR, UK;

p.cullinan@imperial.ac.uk

Accepted 23 April 2015

CrossMark

To cite: Lloyd C, Cullinan P. Thorax 2015;70:581-584.

\section{BASIC SCIENCE}

Another good year for biomarkers

Identification of a systemic molecular signature facilitating early diagnosis and enabling targeted therapy is the Holy Grail for the investigators of many pulmonary diseases, particularly those with heterogeneous presentation such as chronic obstruction pulmonary disease (COPD) or idiopathic pulmonary fibrosis (IPF). Genome-wide gene expression profiling of large groups of patients has revealed a clear gene signature for elastogenesis during COPD and identified fibulin- 5 as a potential molecular target. ${ }^{1}$ Genetic regulation of gene expression patterns was investigated by integrative genomics which identified cystatin C (CST3) and CD22 as causal genes for airflow obstruction. ${ }^{2}$ The fact that these gene expression patterns in COPD are dynamic during treatment, reflecting disease severity, highlights the potential of this approach for the development of molecular phenotypedriven therapy for COPD. ${ }^{3}$ Similarly, a coordinated approach was used to assess the relationship between gene expression patterns, pathological features and systemic biomarkers to identify indicators of disease severity in patients with IPF. MMP3 and CXCL13 emerged as systemic biomarkers that reflect brochiolisation and lymphoid aggregates and are predicted to be of clinical value as prognostic or surrogate markers of disease severity in these patients. ${ }^{4}$ An alternative method of predicting cancer risk in patients presenting with endobronchial squamous metaplasia is the identification of DNA copy number aberration. This technique has proved a highly accurate biomarker for assessment of disease progression and could assist in deciding which patients with endobronchial lesions may benefit from more aggressive treatment or closer surveillance. $^{5}$

\section{Smoking and steroids}

Further evidence that smoking is bad for you-and your immune system-stems from a series of papers examining the effect of cigarette smoke on innate immune function. Tobacco smoke blunted secretion of key innate cytokines such as interferon $\gamma$ and tumour necrosis factor $\alpha$ and impaired the ability of macrophages derived from peripheral blood or the airways to contain mycobacteria within their cell membranes. ${ }^{6}$ This subversion of key innate immune pathways has the potential to increase risk for tuberculosis (TB), particularly in those with underlying pulmonary conditions. In fact, innate immune pathways have been shown to correlate with the severity of stable COPD. ${ }^{7}$ Exposure to cigarette smoke also reduces the effectiveness of steroids, commonly prescribed medications in COPD and asthma. ${ }^{8}$
Although budesonide protects against oxidative stress-induced epithelial dysfunction, cigarette smoke reduces the responsiveness of pulmonary epithelial cells to budesonide, perhaps explaining why steroids do not work in many patients. An elegant series of experiments involving human cells and mice exposed to cigarette smoke revealed a novel mechanism whereby transient receptor potential (TRP) and pannexin channels contribute to smoketriggered ATP release. ${ }^{9}$ These TRP channels have also been shown to be important in mediating virus-induced cough, thus increasing their attractiveness as potential novel therapeutic targets in COPD and other respiratory diseases. ${ }^{10}$

\section{Innate immune mechanisms}

Innate immunity is vital for host defence, and dysfunction in components of this immune network can lead to enhanced pathogen growth and increased inflammation. IL-27 is critical for the control of sepsis-induced impairment of lung antibacterial host defence. Mice deficient in IL-27 were more resistant to secondary bacterial pneumonia and showed significantly lower bacterial burdens and improved survival, leading to the suggestion that manipulation of the IL-27 pathway has therapeutic potential. ${ }^{11}$ Neutrophils provide host immunity to Streptococcus pneumonia via a number of pathways. However, a series of studies in patients with community-acquired pneumonia and mechanistic studies in a mouse model have revealed that one such pathway has been subverted by the bacteria to facilitate bacterial growth rather than clearance. ${ }^{12}$ In contrast to previous reports which outline a host protective role for the neutrophil-derived myeloid related protein (MRP) $8 / 14$, Achouiti and colleagues determined that patients with community-acquired pneumonia (CAP) showed high levels of MRP 8/14 and that mice deficient in MRP14 exhibited reduced bacterial growth and improved survival-a prime example of how a bacterium can manipulate the immune system for its own benefit.

Resident lung cells such as epithelial cells and alveolar macrophages provide vital defence against pathogens in the airways and the consequence of impaired function was demonstrated in exacerbationprone COPD patients. Macrophage responses from these patients were suboptimal in terms of cytokine production and TLR signalling on exposure to respiratory pathogens when compared to macrophages donated from non-exacerbation-prone COPD patients. ${ }^{13}$ Although previous studies have implied that rhinovirus-induced interferon production from epithelial cells is suboptimal in patients with asthma, a new study has shown that defective production of 
type I and III interferons during rhinovirus infection is not universal but rather may be a facet of asthma control. ${ }^{14}$

\section{Manipulation of the immune system}

A sizeable proportion of the space in the Basic Science section of this journal is taken up by studies which use in vivo and in vitro systems to assess the efficacy of potential therapies. Manipulation of immune pathways to improve diseases is the goal for many researchers, particularly those who seek to promote tolerance to common aeroallergens such as pollen. Investigation into how the immune system reacts to Timothy grass identified an immunodominant epitope that promoted reduced $\mathrm{T}$ cell responses when delivered as a peptide in vivo in a mouse model. ${ }^{15}$ Importantly, the epitope was conserved across a range of grasses. Sustained suppression of ragweed-induced allergic inflammation was also achieved using a limited regimen of $\mathrm{CpG}$-containing oligodeoxynucleotide-known to be potent inhibitors of Th2-mediated allergic responses. ${ }^{16}$ However, controlling the immune system may not be as simple as shifting from a Th2 response. Exposure to farm environments has been shown to protect against a Th2 response and the development of allergy, but Robbe and colleagues determined in a mouse model and in agricultural workers that chronic farm exposure shifted T cell response away from a Th2 towards a Th17 phenotype, perhaps increasing the risk for other chronic respiratory conditions, which wins the Thorax Bronze Medal. ${ }^{17}$

There is increasing recognition of the role that proteases play in regulating immune responses. Imbalance in protease networks has been associated with a range of different pulmonary conditions, particularly those where tissue remodelling is a component of the pathology. Increased alveolar apoptosis has been linked to proteolysis via protease-mediated ectodomain shedding of lung epithelial cell adhesion molecule 1 (CADM1). ${ }^{18}$ Targeting particular protease pathways is a potential avenue for therapy. Protease activated receptor (PAR)-1 is a G-protein coupled receptor and interacts with several protease systems involved in tissue remodelling, such as thrombin and members of the matrix metalloproteinase family. A specific PAR-1 antagonist was found to ameliorate the features of bleomycin-induced fibrosis, even when delivered in a therapeutic regimen after fibrosis was established. ${ }^{19}$

\section{Genes and stem cells}

Innovative strategies and systems to manipulate disease progression are vital to determine the in vivo function of particular molecules and gauge the effect on physiological function and disease outcome. Gene manipulation in vivo can be achieved using delivery via viral vectors-an approach which has led to successful reduction of MCP-1 and structural remodelling in a chronic heart failure model. ${ }^{20}$ Similarly, human mesenchymal stem cells (MSCs) engineered to express tumour necrosis factorrelated apoptosis-inducing ligand (TRAIL) successfully incorporate into malignant pleural mesothelioma and induce cancer cell death. $^{21}$ MSCs were tracked in vivo by bioluminescent and fluorescent imaging to delineate the location of the cells delivered to the lungs, highlighting the need for systemic rather than topical application of cells.

\section{Mice, horses and men}

Mouse models are arguably invaluable for the development and testing of hypotheses and potential therapeutic pathways. However, they incur the inevitable criticism of how they relate to human disease. Investigators have tried to mitigate these criticisms by developing models of disease in larger animals: in sheep, whose lungs are more similar to those of humans, and in horses which naturally develop a form of asthma. ${ }^{22}$ However, particular emphasis should be given to innovative ways of investigating disease mechanisms in humans. A study employed autofluorescent bronchoscopy to track the migration of cancerous cells and, together with molecular profiling of biopsies, monitored lesions longitudinally, documenting histological and molecular relationships and winning the Thorax Silver Medal. ${ }^{23}$ Using this combined approach, the authors determined that cancerous cells were able to migrate across histologically normal epithelium to establish new clonal lesions. Summers and colleagues have used gamma scintigraphy to track the migration of ${ }^{111}$ indium-labelled neutrophils through the lungs of healthy volunteers and are our Thorax Gold Medal winners. ${ }^{24}$ They determined that the healthy human lung is able to retain primed neutrophils, and facilitate their depriming and release into the general circulation. This mechanism appeared to be defective in patients with acute respiratory distress syndrome which results in remote organs being exposed to primed neutrophils, contributing to organ damage.

\section{EPIDEMIOLOGY}

One might think that people who ride bicycles or own allotments are by nature gentle souls. Anyone who cycles through London or has presented a clutch of runner beans to the autumn show will tell you that this is not the case; faction and rivalry are rife. So it is in epidemiology, surely the most placid of medical sciences but one where dispute and dogma are rarely far from the surface. We witnessed a little of this in the journal in 2014 with several papers and a robust correspondence on the epidemiology of COPD. It kicked off with an analysis of ethnic differences-across a narrow range of self-reported 'ethnicities'-in respiratory 'impairment' in several thousand North Americans recruited to the NHANES study. ${ }^{25}$ Particular concern was expressed over African Americans who, the authors believe, have especially high rates of respiratory impairment (particularly of a 'restrictive' pattern) associated with mortality (but not respiratory symptoms). Not so, claim their detractors, who elsewhere objected to the notion that ethnic differences in lung volumes are "hard wired into the $\mathrm{DNA}^{26}$ and maintain that the prognostic significance of a given FVC value is independent of ethnic group and that all groups are equally disadvantaged by a low value. The global associations of smoking and poverty with 'COPD' mortality were strikingly displayed by the same Burden of Obstructive Lung Disease (BOLD) study authors in a survey of several thousand adults in 22 centres across the world; ${ }^{27}$ the authors report that in low-income countries, poverty (rather than smoking) dominates the risk of COPD mortality even though spirometrically measured airflow obstruction is overwhelmingly associated with smoking in all regions. There is, they soberly conclude, a serious danger that were a smoking epidemic to become established in these vulnerable regions, it would have even more devastating effects than we have seen so far in the more affluent countries.

Temperatures were similarly raised by the unending dispute over how best to measure abnormality in spirometry. In May the COPDGene investigators reported ${ }^{28}$ that a fixed threshold for $\mathrm{FEV}_{1} / \mathrm{FVC}$ correlated better with a CT-based measure of emphysema than did a 5\% LLN which, the authors reported, failed to identify a population of patients with significant disease. Not so, claimed a correspondent, ${ }^{29}$ since CT densitybased measures of emphysema fail to take into account the growth in air spaces with age and the discordance between LLN and fixed ratios varies by age. This is doubtless a debate that 
will continue, although we wish it wouldn't; the LLN approach is derived from a statistical approach that is otherwise universally accepted for defining normal values within populations.

More stimulating, and infinitely more tragic, were the findings of two related papers from South Korea. The first described a case series ${ }^{30}$ of young adults with a devastating acute lung injury; the second ${ }^{31}$ the epidemiological investigation into its cause. The story began in early 2011 with the admission into the intensive care unit of a Seoul hospital of a cluster of patients with unaccountable respiratory failure. Identification of similar cases soon indicated a strong seasonal pattern of onset and a predilection for young women and their children. The South Korean Center for Disease Control and Prevention and colleagues-to whom we award this year's Thorax Gold Medal for epidemiology_mounted a classic outbreak investigation which suggested very strongly that the cause was inhalation of a particular disinfectant used in home humidifying systems and sold only in that country. That their surmise was correct was established by the absence of any new cases once the product had been withdrawn from sale. We were honoured to commission an editorial ${ }^{32}$ from an eminence grise who, 25 years ago, predicted such an event. A sober reminder that the roots of much -most?-respiratory disease lies in the air we breathe.

And perhaps nowhere more so than in the field of outdoor air pollution, but where should regulators focus their attention? On the (trans-)national control of regional pollutants or more locally on traffic control? A report from the Californian Health Study ${ }^{33}$ where, as it happens, pollution exposures at any range are strongly determined by road traffic, suggests that 'near-road' and regional exposures have adverse effects that are independent of one another. An accompanying editorial ${ }^{34}$ warned that these findings may not be generalisable and called for more studies that use both fixed-site and more local exposure estimates to better disentangle the effects. On the same topic we were delighted to publish a systematic review ${ }^{35}$ of the adverse effects of $\mathrm{PM}_{2.5}$ pollution ('it's bad for you')—a gargantuan effort and a thoroughly 'worthy' paper. Nor did we ignore the indoor; in June we published a manuscript on the risks of cooking with gas. ${ }^{36}$ No overall effect on bronchial responsiveness was found, but those with a null GSTM1 genotype were more responsive if they cooked with gas, especially if they use bottled gas or a stove with both a gas hob and an oven or if their kitchen was unventilated. Curiously (or perhaps not) the effect was the same in men and women.

The Thorax Silver Medal for epidemiology in 2014 goes to a manuscript on the effects of BCG in Greenland. ${ }^{37}$ In 1955, neonatal BCG vaccination was introduced to the national childhood vaccination programme; in 1991 it was removed because the incidence of TB there had fallen so dramatically, only, in 1997, for it to be re-introduced when the pattern reversed. Taking advantage of this quasi-experiment, the investigators estimated that in this setting BCG reduces the incidence of tuberculous infection (and disease) by a remarkable $50 \%$. We thought this was a very fine piece of work, although recognise that the findings are probably not generalisable; the Inuit population of Greenland has very low frequencies of non-tuberculous infection and immunodeficiency, both factors that are thought to reduce the efficiency of BCG.

Other papers that took our fancy but sometimes confused us further-but which nonetheless we were delighted to publishincluded (yet) another chapter in the vitamin D saga. A report from the Copenhagen City Heart Study ${ }^{38}$ suggested that low levels of the magic vitamin are associated with more rapid declines in lung function. We wonder whether we will ever see the observational evidence on vitamin D (too little is bad) reconciled with that from the trials (more doesn't make any difference). In Ecuador (we can't be accused of not getting out enough) researchers failed to observe the urban-rural gradient in childhood atopy and respiratory allergy ${ }^{39}$ that is so readily apparent in many European populations; no, we're not sure what to make of this either. More straightforwardly, and fascinatingly, a survey of adults with pneumococcal pneumonia in the $\mathrm{UK}^{40}$ suggested that close contact with a child was a risk factor (we knew that already) but that if the child was vaccinated then the adult has a significantly reduced risk of vaccine-serotype pneumonia; this, as far as we know, is the first demonstration of herd immunity at an individual level. Finally, an example of an old-fashioned case-control study reported an association between a pro-thrombotic state and incident $\mathrm{IPF}^{41}$ and that being thrombus-prone is associated with more severe disease and earlier mortality. A nice piece of work even if its brave conclusion ('we firmly establish the clotting cascade at the centre of the aetiology and prognosis in IPF') made us raise our eyebrows a little.

Finally, two on mesothelioma. The first, from Australia, ${ }^{42}$ confirmed our belief that the risks of the disease in relation to asbestos level off some 40 or 50 years after exposure, although there are by then precious few people to benefit. The second, from France, ${ }^{43}$ addressed the very difficult but crucial question of why in a high proportion of women it is so difficult to attribute the disease to asbestos exposure. There is growing concern that the explanation lies in there having been sufficient amphibole exposure in European domestic environments to pose a real risk; if this is true, then the implications are very serious indeed. For reminding us of it we are happy to award this manuscript the 2014 Thorax Bronze Medal for epidemiology.

Competing interests None declared.

Provenance and peer review Not commissioned; internally peer reviewed.

\section{REFERENCES}

1 Brandsma CA, van den Berge M, Postma DS, et al. A large lung gene expression study identifying fibulin-5 as a novel player in tissue repair in COPD. Thorax 2015; 70:21-32

2 Lamontagne M, Timens W, Hao K, et al. Genetic regulation of gene expression in the lung identifies CST3 and CD22 as potential causal genes for airflow obstruction. Thorax 2014;69:997-1004.

3 van den Berge M, Steiling K, Timens W, et al. Airway gene expression in COPD is dynamic with inhaled corticosteroid treatment and reflects biological pathways associated with disease activity. Thorax 2014;69:14-23.

4 DePianto DJ, Chandriani S, Abbas AR, et al. Heterogeneous gene expression signatures correspond to distinct lung pathologies and biomarkers of disease severity in idiopathic pulmonary fibrosis. Thorax 2015;70:48-56.

5 Bowcock AM. DNA copy number changes as diagnostic tools for lung cancer. Thorax 2014;69:495-6.

6 van Zyl-Smit RN, Binder A, Meldau R, et al. Cigarette smoke impairs cytokine responses and BCG containment in alveolar macrophages. Thorax 2014;69:363-70.

7 Di Stefano A, Caramori G, Barczyk A, et al. Innate immunity but not NLRP3 inflammasome activation correlates with severity of stable COPD. Thorax 2014;69:516-24

8 Heijink I, van Oosterhout A, Kliphuis N, et al. Oxidant-induced corticosteroid unresponsiveness in human bronchial epithelial cells. Thorax 2014;69:5-13.

9 Baxter M, Eltom S, Dekkak B, et al. Role of transient receptor potential and pannexin channels in cigarette smoke-triggered ATP release in the lung. Thorax 2014;69:1080-9.

10 Abdullah $\mathrm{H}$, Heaney LG, Cosby SL, et al. Rhinovirus upregulates transient receptor potential channels in a human neuronal cell line: implications for respiratory virus-induced cough reflex sensitivity. Thorax 2014;69:46-54.

11 Cao J, Xu F, Lin S, et al. IL-27 controls sepsis-induced impairment of lung antibacterial host defence. Thorax 2014;69:926-37.

12 Achouiti A, Vogl T, Endeman $H$, et al. Myeloid-related protein-8/14 facilitates bacterial growth during pneumococcal pneumonia. Thorax 2014;69:1034-42. 
13 Berenson CS, Kruzel RL, Eberhardt E, et al. Impaired innate immune alveolar macrophage response and the predilection for COPD exacerbations. Thorax 2014;69:811-18.

14 Sykes A, Macintyre J, Edwards MR, et al. Rhinovirus-induced interferon production is not deficient in well controlled asthma. Thorax 2015;69:240-6.

15 Till SJ, Raynsford EJ, Reynolds CJ, et al. Peptide-induced immune regulation by a promiscuous and immunodominant CD4T-cell epitope of Timothy grass pollen: a role of Cbl-b and Itch in regulation. Thorax 2014;69:335-45.

16 Campbell JD, Kell SA, Kozy HM, et al. A limited CpG-containing oligodeoxynucleotide therapy regimen induces sustained suppression of allergic airway inflammation in mice. Thorax 2014;69:565-73.

17 Robbe P, Spierenburg EA, Draijer C, et al. Shifted T-cell polarisation after agricultural dust exposure in mice and men. Thorax 2014;69:630-7.

18 Mimae T, Hagiyama M, Inoue T, et al. Increased ectodomain shedding of lung epithelial cell adhesion molecule 1 as a cause of increased alveolar cell apoptosis in emphysema. Thorax 2014;69:223-31.

19 Lin C, Duitman J, Daalhuisen J, et al. Targeting protease activated receptor-1 with P1pal-12 limits bleomycin-induced pulmonary fibrosis. Thorax 2014;69:152-60.

20 Park JE, Lyon AR, Shao D, et al. Pulmonary venous hypertension and mechanical strain stimulate monocyte chemoattractant protein-1 release and structural remodelling of the lung in human and rodent chronic heart failure models. Thorax 2014;69:1120-7.

21 Sage EK, Kolluri KK, McNulty K, et al. Systemic but not topical TRAlL-expressing mesenchymal stem cells reduce tumour growth in malignant mesothelioma. Thorax 2014;69:638-47.

22 Boivin R, Vargas A, Lefebvre-Lavoie J, et al. Inhaled corticosteroids modulate the (+)insert smooth muscle myosin heavy chain in the equine asthmatic airways. Thorax 2014;69:1113-19.

23 Pipinikas CP, Kiropoulos TS, Teixeira VH, et al. Cell migration leads to spatially distinct but clonally related airway cancer precursors. Thorax 2014;69:548-57.

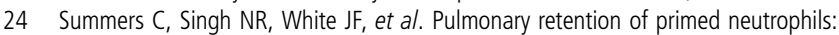
a novel protective host response, which is impaired in the acute respiratory distress syndrome. Thorax 2014;69:623-9.

25 Vaz Fragoso CA, McAvay G, Gill TM, et al. Ethnic differences in respiratory impairment. Thorax 2013;69:55-62.

26 Burney $\mathrm{P}$, Kato B, Janson $\mathrm{C}$, et al, Burden of Obstructive Lung Disease (BOLD) Study. Chronic obstructive pulmonary disease mortality and prevalence: the associations with smoking and poverty: a BOLD analysis-authors' reply. Thorax 2014;69:869-70.

27 Burney $\mathrm{P}$, Jithoo A, Kato B, et al. Chronic obstructive pulmonary disease mortality and prevalence: the associations with smoking and poverty - $\mathrm{a}$ BOLD analysis. Thorax 2014;69:465-73.
28 Bhatt SP, Sieren JC, Dransfield MT, et al. Comparison of spirometric thresholds in diagnosing smoking-related airflow obstruction. Thorax 2014;69:409-14.

29 Miller MR. Comparison of spirometric thresholds in diagnosing smoking-related airflow obstruction. Thorax 2014;69:1145-6.

30 Hong SB, Kim HJ, Huh JW, et al. A cluster of lung injury associated with home humidifier use: clinical, radiological and pathological description of a new syndrome. Thorax 2014;69:694-702.

31 Kim HJ, Lee MS, Hong SB, et al. A cluster of lung injury cases associated with home humidifier use: an epidemiological investigation. Thorax 2014;69:703-8.

32 Pickering CAC. Humidifiers: the use of biocides and lung disease. Thorax 2014;89:692-3

33 Urman R, McConnell R, Islam T, et al. Associations of children's lung function with ambient air pollution: joint effects of regional and near-roadway pollutants. Thorax 2014;69:540-7

34 Kunzli N. Effects of near-road and regional air pollution: the challenge of separation. Thorax 2014;69:503-4.

35 Atkinson RW, Kang S, Anderson HR, et al. Epidemiological time series studies of PM2.5 and daily mortality and hospital admissions: a systematic review and meta-analysis. Thorax 2014;69:660-5.

36 Amaral AF, Ramasamy A, Castro-Giner $F$, et al. Interaction between gas cooking and GSTM1 null genotype in bronchial responsiveness: results from the European Community Respiratory Health Survey. Thorax 2014;69:558-64.

37 Michelsen SW, Soborg B, Koch A, et al. The effectiveness of BCG vaccination in preventing Mycobacterium tuberculosis infection and disease in Greenland. Thorax 2014;69:851-6.

38 Afzal S, Lange P, Bojesen SE, et al. Plasma 25-hydroxyvitamin D, lung function and risk of chronic obstructive pulmonary disease. Thorax 2014;69:24-31.

39 Cooper PJ, Vaca M, Rodriguez A, et al. Hygiene, atopy and wheeze-eczema-rhinitis symptoms in schoolchildren from urban and rural Ecuador. Thorax 2014;69:232-9.

40 Rodrigo C, Bewick T, Sheppard C, et al. Pneumococcal serotypes in adult non-invasive and invasive pneumonia in relation to child contact and child vaccination status. Thorax 2014;69:168-73.

41 Navaratnam V, Fogarty AW, McKeever T, et al. Presence of a prothrombotic state in people with idiopathic pulmonary fibrosis: a population-based case-control study. Thorax 2014;69:207-15.

42 Reid $\mathrm{A}$, de Klerk NH, Magnani C, et al. Mesothelioma risk after 40 years since first exposure to asbestos: a pooled analysis. Thorax 2014;69:843-50.

43 Lacourt A, Gramond C, Rolland P, et al. Occupational and non-occupational attributable risk of asbestos exposure for malignant pleural mesothelioma. Thorax 2014;69:532-9. 